\title{
Epicutaneous immunization with protein antigen TNP-Ig alleviates TNBS-induced colitis in mice
}

\author{
Monika Majewska-Szczepanik, Marta Góralska, Katarzyna Marcińska, \\ Magdalena Zemelka-Wiącek, Anna Strzępa, Iwona Dorożyńska, \\ Marian Szczepanik
}

Department of Medical Biology, Jagiellonian University College of Medicine, Kopernika 7, PL 31-034 Kraków, Poland

Correspondence: Marian Szczepanik, e-mail: mmszczep@cyf-kr.edu.pl

\begin{abstract}
:
Background: Ulcerative colitis (UC) is a chronic inflammatory autoimmune disease with limited treatment modalities. The animal model of colitis induced by treatment with trinitrobenzene sulfonic acid (TNBS-colitis) is commonly used to test new therapies of this disease. In our previous work we found that epicutaneous (EC) immunization with protein antigen induced a state of profound immunosuppression that inhibited inflammatory response in contact sensitivity, in experimental autoimmune encephalomyelitis (EAE) and in allogeneic skin graft rejection.

Methods: TNBS-induced colitis was used as an experimental model.

Results: In our current work, we showed that EC immunization with TNP-conjugated mouse immunoglobulin (TNP-Ig) prior to induction of TNBS-colitis alleviates disease severity what was determined by the body weight, the length and the weight of the colon, the histological activity index (HAI) and myeloperoxidase activity (MPO). Observed amelioration of the disease in TNP-Ig patched mice was accompanied with decreased production of IFN- $\gamma$ and IL-17A by splenocytes. Additionally, spleen cells isolated from mice EC immunized with TNP-Ig prior to colitis induction showed increased production of IL-10 suggesting that this cytokine might be involved in inhibiting inflammatory response in the colon.

Conclusion: This work shows that EC immunization with protein antigen prior to TNBS-colitis induction ameliorates disease and observed suppression of inflammatory response in the colon might be mediated by IL-10.
\end{abstract}

Key words:

inflammatory bowel disease, ulcerative colitis, TNBS-colitis, supression

\begin{abstract}
Abbreviations: CD - Crohn's disease, CIA - collagen induced arthritis, CS - contact sensitivity, DNFB - dinitrofluorobenzene, DNP - 2,4-dinitrophenol, EAE - experimental autoimmune encephalomyelitis, EC - epicutaneous, FCS - fetal calf serum, HAI - histological activity index, IBD - inflammatory bowel disease, IFN- $\gamma$ - interferon gamma, Ig - immunoglobulins, MBP - myelin basic protein, MLN - mesenteric lymph nodes, MPO - myeloperoxidase, SPF - specific pathogen-free, Th1 - T helper 1, Th2 - T helper 2, TNBSA - 2,4,6- trinitrobenzene sulfonic acid, TNP-Cl - 2,4,6-trinitrophenyl chloride, TNP-Ig - TNP conjugated mouse immunoglobulins, Ts - T suppressor cell, UC - ulcerative colitis
\end{abstract}

\section{Introduction}

Inflammatory bowel disease (IBD) in humans, such as ulcerative colitis (UC) and Crohn's disease (CD), is a complex chronic inflammatory disease of largely unknown cause and affects 3.6 million people in Europe and the USA, mainly of Caucasian descent [6]. The multifactorial mechanisms underlining IBD are emphasized by the large number of host IBD sus- 
ceptibility genes that have been characterized in recent years [3]. The contribution of the host immune system and genetic factors that predispose to IBD have been extensively investigated. At present, it is believed that IBD relates to genetic predisposition, an environmental trigger and an aberrant immune reaction [12].

The immune response reflects defects in both innate and adaptive immunity. Defects of the innate immune response lead to inappropriate responses to commensal gut flora including the production of various cytokines such as IL-12 and IL-23 or IL-13 resulting in either Th1/Th17 or Th2 responses [12].

Pathogenesis of IBD is not fully understood but it is well accepted that CD is Th1/Th17-T cell-driven process whereas UC is Th2-like T-cell-driven process [19].

To study pathogenesis of human diseases and to test new therapeutics animal models are used. One of the models that mimic UC can be induced in susceptible mouse strains by intrarectal instillation of the haptens such as TNBS or oxazolone dissolved in ethanol with or without skin preimmunization [27]. Ethanol is required to damage the mucosal barrier, whereas the hapten is believed to conjugate with colonic self or microbiota proteins. It was previously shown that TNBS-induced IBD in SJL mice mimics colitis characterized by a predominant Th1-mediated immune response with dense infiltration of lymphocytes/macrophages and thickening of the colon wall $[13,27]$.

Currently used medical therapy of IBD consists of salicylates, corticosteroids, immunosuppressants and immunomodulators. However, their use may result in severe side effects and complications, such as an increased rate of malignancies or infectious diseases [5, 15]. For many years there have been numerous efforts to find a new effective method that would allow controlling specifically unwanted immune responses that occur during autoimmune reaction.

Our previous work employing Th1-mediated contact sensitivity (CS) to TNP showed that epicutaneous (EC) immunization with TNP-Ig (TNP-conjugated mouse immunoglobulins) prior to skin sensitization with TNP-Cl strongly suppressed CS [17]. Observed inhibition of CS was mediated by TCR $\alpha \beta^{+} \mathrm{CD} 4^{+} \mathrm{CD}^{+}$double positive T suppressor (Ts) cells that inhibit sensitization and elicitation of CS-effector T cell responses via TGF- $\beta$ [8].

In the current work we showed that maneuver of EC immunization with TNP-Ig prior to UC induction alleviates disease severity what was determined by macroscopic and microscopic evaluation confirmed by the measurement of myeloperoxidase (MPO) activity and cytokine production.

\section{Materials and Methods}

\section{Mice}

Specific pathogen-free (SPF) female SJL $\left(\mathrm{H}-2^{\mathrm{s}}\right)$ mice from the breeding unit of the Department of Medical Biology, Jagiellonian University College of Medicine were used. Mice were rested for at least 1 week before use, maintained under SPF conditions, and used at $6-10$ weeks of age in groups of 10-14. All experiments were conducted according to guidelines of Local Ethical Commission No. 71/2010.

\section{Reagents}

2,4,6-trinitrobenzene sulfonic acid (TNBSA), hexadecyltrimethylammonium bromide and o-dianisidine dihydrochloride were obtained from Sigma (St. Louis, MO, USA). 2,4,6-trinitrophenyl chloride (TNP-Cl, picryl chloride) from Nacalai Tesque (Kyoto, Japan) was recrystallized twice and stored protected from light. RPMI 1640 and fetal calf serum (FCS) were from Life Technologies (Grand Island, NY, USA). Mouse immunoglobulins (Ig) were prepared from $\mathrm{CBA} / \mathrm{J}$ mouse sera and conjugated with TNP hapten [25, 26]. A single preparation with the level of substitution of 40 TNP per Ig molecule $\left(\mathrm{TNP}_{40}-\mathrm{Ig}\right)$ was used throughout.

\section{Monoclonal antibodies}

Purified rat anti-mouse TGF- $\beta 1$, and biotinylated antimouse, anti-human, anti-pig TGF- $\beta 1$ antibodies were obtained from BD Biosciences (San Diego, CA, USA).

\section{Induction of TNBS-colitis}

Mice were actively sensitized by topical application of $0.15 \mathrm{ml}$ of $5 \% \mathrm{TNP}-\mathrm{Cl}$ in acetone : ethanol mixture $(1: 3, v / v)$ to the shaved abdomen on day " 0 ". On day " +7 " the body weight of mice was estimated and animals were fasted for $12 \mathrm{~h}$. Next day (day “+8”) mice were anesthetized and the catheter was carefully inserted into the colon until the tip was $40 \mathrm{~mm}$ approximal to the anus. Then, $100 \mu \mathrm{l}$ of TNBSA $(2.5 \%$ TNBSA in 50\% ethanol) was administered [26]. Control group was sham immunized and then received intrarectal (ir) dose of $100 \mu \mathrm{l}$ of $50 \%$ ethanol. Animals were monitored daily for weight, water/food consumption, morbidity and stool consistency. In some of 
the experiments animals were sacrificed three days after ir TNBSA administration, colons were isolated and then length of the colons was measured. Additionally, the weights of $20 \mathrm{~mm}$ pieces of the colon were measured. Samples of the colon were tested for myeloperoxidase (MPO) activity. Additionally, sections of the colon were stained with hematoxylin and eosin (H\&E) and histological scores were determined.

\section{EC immunization with TNP-Ig to induce suppression}

Mice were shaved on their backs with a razor blade before immunization. On day " -7 " $100 \mu 1$ of either TNP-Ig (at $1 \mathrm{mg} / \mathrm{ml}$ ) in PBS, or PBS alone was applied to gauze in the center of an occlusive patch (DuoDerm Extra Thin, ConvaTec, Princeton, NJ, USA) which was then affixed to their backs [22]. Patches were left intact for 4 days and then replaced with new antigen containing patches and kept on until day " 0 ". On day " 0 " the patches were removed and TNBS-colitis was induced (Scheme 1).

\section{MPO assay}

Neutrophil infiltration to the inflamed colon was indirectly quantitated using an MPO assay, as described previously [23]. Colons were removed three days after ir administration of TNBSA and $10 \mathrm{~mm}$ long pieces from the distal site of the colon were taken. The samples were homogenized in $0.5 \%$ hexadecyltrimethylammonium bromide $\mathrm{pH}=6.0(50 \mathrm{mg}$ of tissue $/ \mathrm{ml}$ ). The homogenates were freeze thawed 3 times, centrifuged at $40,000 \times \mathrm{g}$ and then $0.1 \mathrm{ml}$ aliquots were mixed with $2.9 \mathrm{ml}$ phosphate buffer $(\mathrm{pH}=6.0)$ containing $0.167 \mathrm{mg} / \mathrm{ml}$ o-dianisidine dihydrochloride and $5 \times 10^{-4} \% \mathrm{H}_{2} \mathrm{O}_{2}$ and incubated at $25^{\circ} \mathrm{C}$ for $20 \mathrm{~min}$. The absorbance was measured at $460 \mathrm{~nm}$ in 96-well flat bottom plates. MPO activity was expressed in units per protein concentration (U/mg of protein).

\section{Grading of histologic changes}

Mice were sacrificed, distal colon was removed, opened, washed with PBS and spread flat on a paper with mucosa facing up and fixed in $10 \%$ buffered formalin. Tissue was oriented to get perpendicular cuts of rectal wall [23]. Routine tissues processing of paraffin embedding were followed by H\&E staining of $3 \mu \mathrm{m}$ paraffin sections. The following parameters

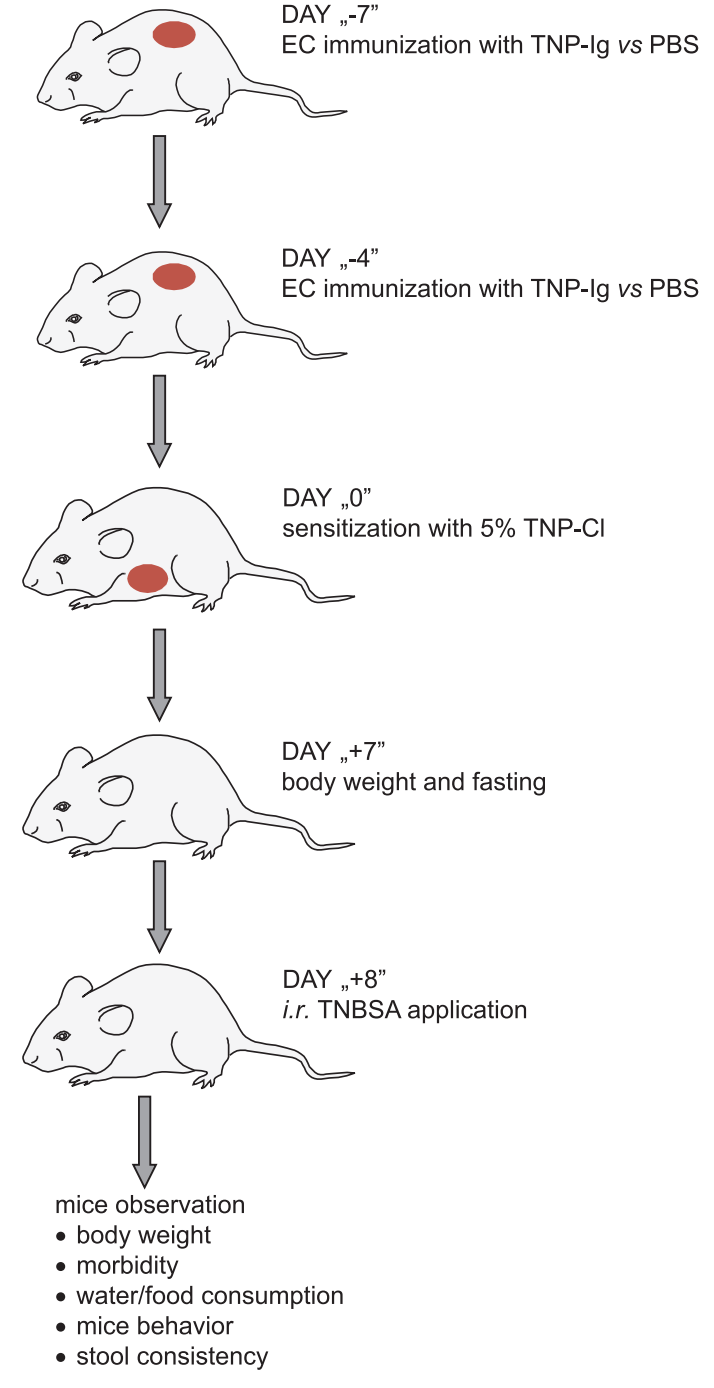

Scheme 1. SJL mice were shaved on their backs before immunization. On day " -7 " mice were epicutaneoulsy (EC) treated with $100 \mu \mathrm{l}$ of either TNP-lg (at $1 \mathrm{mg} / \mathrm{ml}$ ) in PBS, or PBS alone in a form of gauze patch. Patches were left intact for 4 days and then replaced with new antigen containing patches and kept on until day " 0 ". On day " 0 " the patches were removed and TNBS-colitis was induced. To induce colitis, mice were skin sensitized with $0.15 \mathrm{ml}$ of $5 \% \mathrm{TNP}-\mathrm{Cl}$ in acetone : ethanol mixture $(1: 3)$ to the shaved abdomen. On day " +7 " the body weight of mice was measured and animals were fasted for $12 \mathrm{~h}$. Next day (day "+8") mice received intrarectal (ir) dose of $100 \mu$ l of TNBSA (2.5\% TNBSA in 50\% ethanol). Control mice were sham immunized and then received ir $100 \mu \mathrm{l}$ of $50 \%$ ethanol. Animals were monitored daily for weight, water/food consumption, morbidity and stool consistency or were used for in vitro tests

were used for calculation [1]: a) epithelial damage $(0=$ none, $1=$ minimal loss of goblet cells, $2=$ extensive loss of goblet cells, $3=$ minimal loss of crypts and extensive loss of goblet cells, and $4=$ extensive loss of crypts); b) infiltration ( $0=$ none, $1=$ infiltrate around crypt bases, 2 = infiltrate in muscularis mucosa, $3=$ extensive infiltrate in muscularis mucosa with 
edema, and 4 = infiltration of submucosa). The histological activity index (HAI) was calculated as the sum of the epithelium and infiltration score, resulting in the total HAI score ranging from 0 (unaffected) to 8 (severe colitis).

\section{Measurement of cytokines in culture supernatants}

To test if EC immunization with TNP-Ig affects cytokine production in mice with induced colitis, SJL mice were EC exposed to TNP-Ig (Group C) or PBS (Group B) prior to colitis induction. Control mice were sham sensitized and received $i r$ application of ethanol alone (Group A). Three days after ir instillation of either TNBSA (Groups B and C) or ethanol alone (Group A) mice were sacrificed and spleens (SPL) and mesenteric lymph nodes (MLN) were collected.

Spleen or MLN cells $\left(3 \times 10^{6}\right)$ from tested and control groups were cultured in $1 \mathrm{ml}$ RPMI 1640 medium supplemented with 5\% FCS in the presence of 100 $\mu \mathrm{g} / \mathrm{ml}$ TNP-Ig. Cells were distributed in triplicate wells in flat 24 well Falcon plates. After 48 h, culture supernatants were collected and then tested for cytokine concentration [16].

Culture supernatants were tested for IL-4, IL-10, IL$17 \mathrm{~A}, \mathrm{IFN}-\gamma$ and TGF- $\beta$ concentration with the use of BD OptEIA Set (BD Biosciences, San Diego, CA, USA).

For estimating TGF- $\beta$ concentrations, supernatants were first acidified with $1 \mathrm{M} \mathrm{HCl}$ diluted 1:25 and then neutralized with $1 \mathrm{M} \mathrm{NaOH}$ to $7.0 \mathrm{pH}$ in the same proportion. Cytokine concentrations in culture supernatants were measured by sandwich ELISA using monoclonal rat anti-mouse TGF- $\beta$ antibodies as capture antibody and biotinylated monoclonal rat anti-mouse, -human, -pig TGF- $\beta$ antibodies as the secondary antibody. The reaction was developed with horseradish peroxidase streptavidin, followed by o-phenylenediamine and $\mathrm{H}_{2} \mathrm{O}_{2}$ as substrate, and was stopped with $3 \mathrm{M} \mathrm{H}_{2} \mathrm{SO}_{4}$. The optical density of each well was measured in a 96-well plate reader at $492 \mathrm{~nm}$. All determinations were done in triplicate. Standard curve was generated with recombinant mouse TGF- $\beta$. The lower concentration limit was 30 pg/ml TGF- $\beta$.

\section{Statistics}

Data in graphs are shown as the mean \pm SE. ANOVA followed by Student's $t$-test was used for multiple comparisons. Statistical significance was set at $\mathrm{p}<0.05$.

\section{Results}

\section{EC immunization with TNP-Ig prior to colitis induction protects from body weight loss}

SJL mice were epicutaneously immunized with TNPIg (Group C) or PBS (Group B) prior to colitis induction as described in Materials and Methods. Mice in negative control (Group A) were sham sensitized and then received ir ethanol alone. Body weight was monitored daily starting one day before ir instillation of TNBSA or ethanol.

The mean weight of mice before ethanol or TNBSA administration was $25.1 \pm 1.9 \mathrm{~g}$. Data presented in Figure 1 show that animals EC treated with PBS prior to induction of colitis significantly lost body weight when compared to negative control (Group B vs. A) and animals that were EC immunized with TNP-Ig prior disease induction (Group B vs. C). This data suggest that EC immunization with TNP-Ig prior to colitis induction significantly attenuates body weight loss associated with TNBSA induced colitis.

\section{EC immunization with TNP-Ig suppresses inflammatory reaction in the colon}

To investigate the influence of EC immunization with TNP-Ig on inflammatory response in the colon, animals were sacrificed three days after ir TNBSA or ethanol administration. The colons were isolated, measured and then histologic evaluation was per-

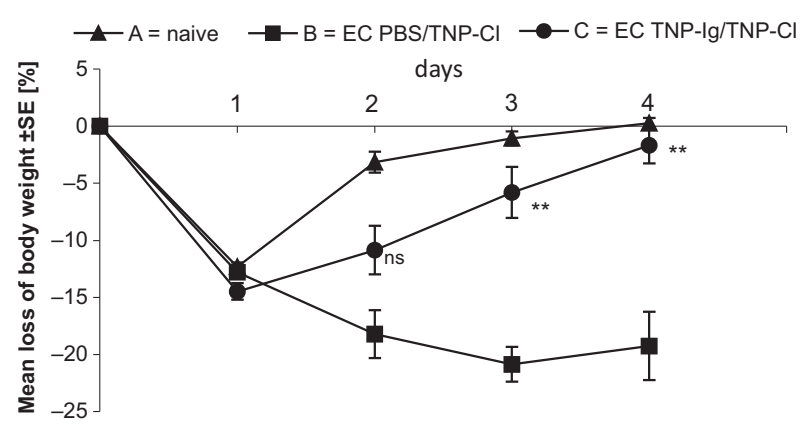

Fig. 1. EC immunization with TNP-Ig prior to colitis induction inhibits body weight loss. SJL mice were EC immunized with $100 \mu \mathrm{l}$ TNP-lg (at $1 \mathrm{mg} / \mathrm{ml}$ ) (Group C) or PBS (Group B) for seven days prior to colitis induction. TNBS-colitis was induced via skin sensitization with $150 \mu \mathrm{l}$ of $5 \%$ TNP-Cl and subsequent ir instillation of $100 \mu \mathrm{l}$ of TNBSA $(2.5 \%$ TNBSA in $50 \%$ ethanol) as described in Materials and Methods. Mice in negative control (Group A) were sham sensitized and then received ir ethanol alone. Body weight was monitored daily. Data represent the mean \pm SE, $n=10-14$ mice per group; ${ }^{* *} p \leq 0.01$ 

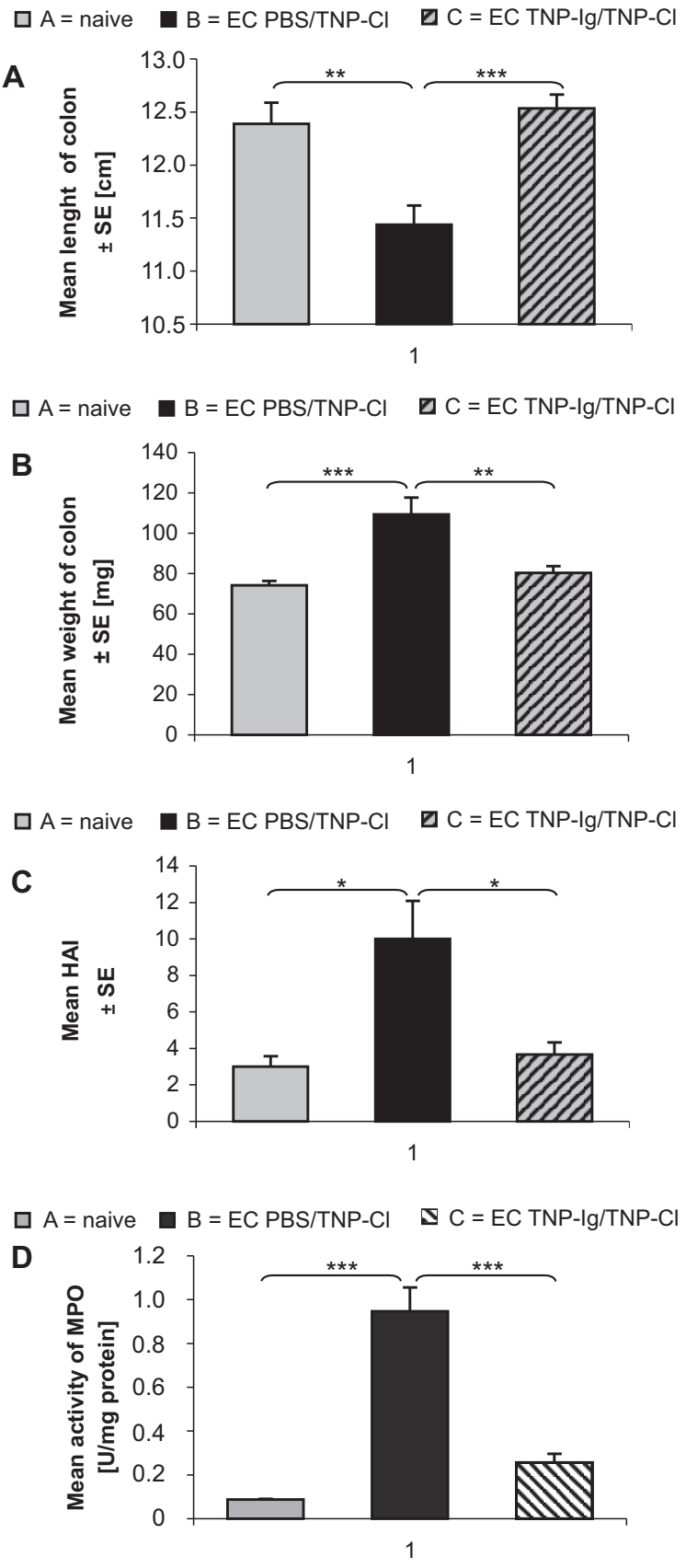

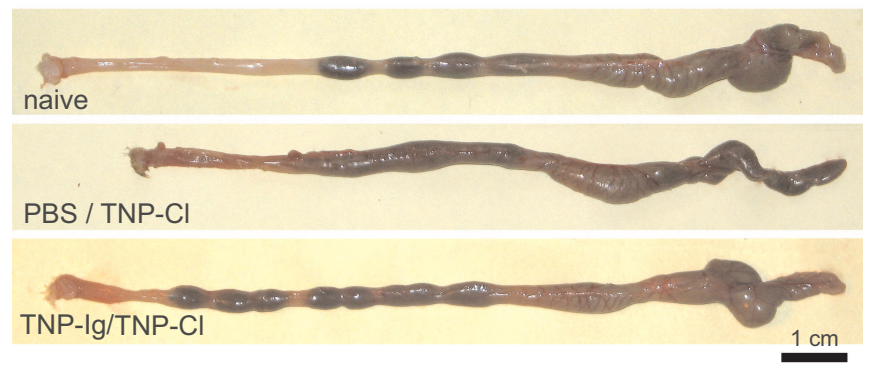

Fig. 2. Skin patching with TNP-Ig alleviates inflammatory reaction in the colon. (A) Influence of EC immunization with TNP-lg prior to colitis induction on colon length. SJL mice were EC immunized with $100 \mu \mathrm{l}$ TNP-Ig (at $1 \mathrm{mg} / \mathrm{ml}$ ) (Group C) or PBS (Group B) for seven days and then patches were removed and colitis was induced as described in Materials and Methods. Mice in negative control (Group A) were sham sensitized and then received ir ethanol alone. Three days after ir TNBSA or ethanol administration, animals were sacrificed, colons were isolated and colon length was measured. Data represent the mean \pm SE, $n=10-14$ mice per group; ${ }^{* *} p \leq 0.01$, ${ }^{* * *} p \leq 0.001$. (B) Effect of EC immunization with TNP-Ig prior to colitis induction on colon weight. Animals were EC treated with TNP-Ig (Group C) or PBS (Group B) for one week and then patches were removed and TNBS-colitis was induced as described in Materials and Methods. The negative control (Group A) consisted of mice that were sham sensitized and then treated ir with ethanol alone. Three days after ir TNBSA or ethanol administration, animals were sacrificed, colons were isolated and colon weight was measured. Data represent the mean $\pm \mathrm{SE}$, $n=10-14$ mice per group; ${ }^{* *} p \leq 0.01,{ }^{* *} p \leq 0.001$. (C) Effect of patching with TNP-lg prior to colitis induction on colon histology. Mice were patched with TNP-Ig (Group C) or PBS (Group B) for seven days prior to colitis induction as described in Materials and Methods. Mice in negative control (Group A) were sham sensitized and then received ir ethanol alone. Three days after ir TNBSA or ethanol application colons were isolated, processed and evaluated for histologic changes as described in Materials and Methods. The histological activity index (HAl) was calculated as the sum of the epithelium damage and infiltration score, resulting in the total HAI score ranging from 0 (unaffected) to 8 (severe colitis). Data represent the mean \pm SE, $n=10-14$ mice per group; * $p \leq 0.05$. (D) Impact of EC treatment with TNP-Ig prior to colitis induction on MPO activity. Animals were EC immunized with TNP-Ig (Group C) or PBS (Group B) for seven days prior to colitis induction and then colitis was induced as described in Materials and Methods. Mice in negative control (Group A) were sham sensitized and then received ir ethanol alone. Three days after ir TNBSA or ethanol instillation colons were isolated, homogenized and tested for MPO activity. Ten millimeters long pieces of colon were homogenized in $0.5 \%$ hexadecyltrimethylammonium bromide. Then, homogenate aliquots were mixed with phosphate buffer containing o-dianisidine dihydrochloride and $\mathrm{H}_{2} \mathrm{O}_{2}$ and incubated at $25^{\circ} \mathrm{C}$ for $20 \mathrm{~min}$. The absorbance was measured at $460 \mathrm{~nm}$. MPO activity was expressed in units per protein concentration (U/mg of protein). Results are shown as the mean \pm SE, $n=10-14$ mice per group; ${ }^{* * *} p \leq 0.001$ formed or samples were homogenized and tested for MPO activity. Data presented in Figure 2A show that animals EC treated with PBS prior to induction of colitis have significantly shorter colons when compared to negative control (Group B vs. A) and mice that were EC immunized with TNP-Ig prior to disease induction (Group C vs. B). Additionally, we found that colon weight of animals EC treated with PBS prior disease in- duction was significantly higher when compared to negative control (Fig. 2B; Group B vs. A) and animals patched with TNP-Ig prior to colitis induction (Fig. 2B; Group $\mathrm{C} v s$. B). These findings were fully confirmed by histologic evaluation (Fig. 2C) and MPO activity in colon homogenates (Fig. 2D). This data indicate that maneuver of EC immunization with TNP-Ig significantly inhibits inflammatory response in the colon. 

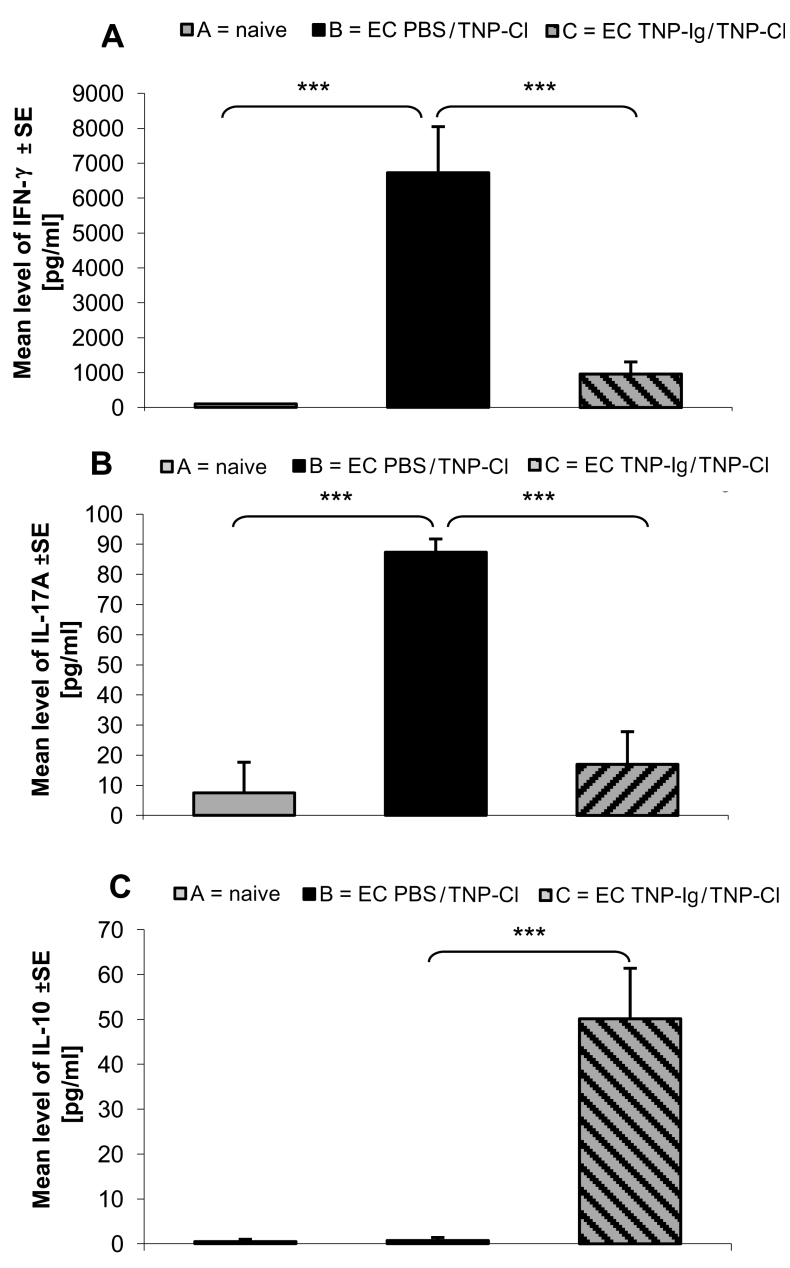

Fig. 3. EC immunization with TNP-Ig prior to colitis induction inhibits IFN- $\gamma$ and IL-17A production. Mice were EC immunized with TNP-Ig (Group C) or PBS (Group B) for 7 days. On day "+7" patches were removed and colitis was induced as described in Materials and Methods Mice in negative control (Group A) were sham sensitized and then received ir ethanol alone. Three days after ir TNBSA application, mice were sacrificed, spleens and mesenteric lymph nodes were collected and processed under aseptic, conditions and then $3 \times 10^{6}$ SPLC or MLNC were cultured in the presence of $100 \mu \mathrm{g} / \mathrm{ml}$ TNP-lg. Cells were distributed in triplicate wells in flat 24 well Falcon plates. After $48 \mathrm{~h}$ culture, supernatants were collected and then tested for concentration of the following cytokines IFN- $\gamma(\mathbf{A})$, IL-17A (B) and IL-10 (C). Results are presented as the mean $\pm \mathrm{SE}, \mathrm{n}=4$; ${ }^{* * *} \mathrm{p} \leq 0.001$

\section{Skin application of TNP-Ig prior to colitis induction inhibits proinflammatory cytokine production}

Mice were patched with TNP-Ig (Group C) or PBS (Group B) for 7 days. On day " +7 " patches were removed and colitis was induced as described in Materials and Methods. Three days after ir TNBSA instillation, mice were sacrificed, spleens were isolated and cell cultures were prepared to evaluate cytokine production. Mice in negative control (Group A) were sham sensitized and then received ir ethanol alone. Data presented in Figure 3A show that EC immunization with TNP-Ig prior to colitis induction significantly inhibits IFN- $\gamma$ (Fig. 3A; Group C vs. B) and IL-17A (Fig. 3B; Group C vs. B) production. Interestingly, production of IFN- $\gamma$ and IL-17A by spleen cells form TNP-Ig patched mice was only slightly higher than production of these cytokines by splenocytes isolated from control mice (Figs. 3A and 3B; Group C vs. A).

To determine the mechanism of observed amelioration of TNBS-colitis induced by EC immunization with TNP-Ig, culture supernatants were additionally tested for the presence of anti-inflammatory cytokines. Data presented in Figure 3C show that EC application of TNP-Ig prior to colitis induction results in increased production of IL-10 by mesenteric lymph nodes (MLN) (Group C vs. B). However, IL-4 and TGF- $\beta$ were undetectable (data not shown).

\section{Discussion}

Our current work showed that maneuver of EC immunization with TNP-Ig prior to UC induction alleviates disease severity what was determined by macroscopic and microscopic evaluation confirmed by the measurement of myeloperoxidase (MPO) activity and cytokine production.

Our previous work employing Th1-mediated CS to TNP showed that EC application of hapten-conjugated protein antigen in the form of gauze patch prior to hapten sensitization significantly suppressed CS in mice $[17,22]$. Then, we found that EC immunization with myelin basic protein (MBP) prior to induction of experimental model of multiple sclerosis (EAE) resulted in protection from developing disease $[9,24$, 25]. More recently, we showed that EC immunization with protein antigen suppresses Tc1-dependent and NK cell mediated CS to DNP $[10,11]$ and an animal model of skin graft rejection [8]. Then, experiments in an animal model of arthritis [collagen induced arthritis (CIA)] showed that EC immunization with collagen prior to CIA induction alleviates disease [21]. Finally, our clinical study proved for the first time that EC application of myelin peptides generates tolerogenic responses and attenuates autoimmunity in multiple sclerosis patients [7]. 
This let us to speculate that maneuver of EC immunization with a proper antigen might become an universal method to induce tolerance that could control unwanted immune responses. To verify this hypothesis we tested efficacy of skin-induced suppression in animal model of colitis ulcerosa.

As an experimental model we used mouse model of TNBS-induced colitis [26]. Data presented in Figure 1 show that SJL mice EC immunized with TNP-Ig slightly lost body weight after ir instillation of TNBSA when compared to control group (mice sham sensitized and treated $i r$ with ethanol alone) (Group C vs. A). Moreover, EC application of TNP-Ig prior to colitis induction significantly protected mice from body weight loss when compared to animals EC treated with PBS before disease induction (Group C $v s$. B). This data might suggest that similarly to other models [8-11, 17, 20, 21, 22, 24, 25] EC immunization with protein antigen induces tolerance that inhibits inflammatory response in the colon. To confirm our speculation in a separate set of experiments, colon length, colon histology and MPO activity in tissue homogenates were evaluated. All three parameters showed that EC immunization with TNP-Ig prior disease induction significantly ameliorates inflammatory response in the colon (Figs. 2A-D; Group C vs. B).

It is commonly well accepted that $\mathrm{CD}$ is Th1/ Th17-T cell-driven process whereas UC is Th2-like T-cell-driven process [19]. On the other hand, it was already shown that TNBS-colitis exhibits heighten Th1-Th17 response [1]. To test if observed amelioration of colitis in mice EC treated with TNP-Ig is related to the suppression of Th1 and Th17 responses, we evaluated production of proinflammatory cytokines in culture supernatants. Data presented in Figure 3 show that EC immunization with TNP-Ig prior to colitis induction significantly inhibits IFN- $\gamma$ and IL-17A production when compared to mice EC treated with PBS before disease induction (Fig. 3A; Group C vs. A and Fig. 3B Group C vs. A, respectively).

It is well accepted that anti-inflammatory cytokines produced by $\mathrm{Th} 2$ and $\mathrm{T}$ regulatory cells can inhibit inflammatory response mediated by either Th1 or Th17 lymphocytes [2, 14, 18, 21].

To determine whether skin-induced suppression that ameliorates TNBS-colitis is mediated by antiinflammatory cytokines, culture supernatants produced by splenocytes isolated from mice EC treated with TNP-Ig or PBS prior to colitis induction were tested for the presence of IL-4, IL-10 and TGF- $\beta$. Data presented in Figure 3C showed that EC immunization with TNP-Ig prior to disease induction results in increased production of IL-10 by MLN when compared with animals that were EC treated with PBS before colitis induction. IL-10 itself functions as an anti-inflammatory cytokine, and limits excessive tissue disruption caused by inflammation. Thus, it is possible that observed amelioration of the disease in our system after skin patching with TNP-Ig is partly mediated by IL-10. Our finding is in line with report from other laboratories showing that indeed IL-10 plays an important role in controlling inflammatory response during TNBS-colitis [4, 28]. However, other sources of these cytokine are possible. Further experiments are required to determine the source of antiinflammatory cytokines in our system.

In summary, this work shows that EC immunization with protein antigen prior to TNBS-colitis induction ameliorates disease and observed suppression of inflammatory response in the colon might be mediated by IL-10.

\section{Acknowledgments:}

This work was supported by grants from Ministry of Science and Higher Education N N401 3553 33, N N401 006939, N N401 545940, UMO-2011/01/B/NZ6/00300 and K/ZDS/002182 to Marian Szczepanik, and N N401 000936 and K/ZDS/003673 to Monika Majewska-Szczepanik.

\section{References:}

1. Alex P, Zachos NC, Nguen T, Gonzales L, Chen T-E, Conklin LS, Centola M, Li X: Distinct cytokine patterns identified from multiplex profiles of murine DSS and TNBS-induced colitis. Inflamm Bowel Dis, 2009, 15, 341-352.

2. Awasthi A, Murugaiyan G, Kuchroo VK: Interplay between effector Th17 and regulatory cells. J Clin Immunol, 2008, 28, 660-670.

3. Cho JH, Brant SR: Recent insights into the genetics of inflammatory bowel disease. Gastroenterology, 2011, 140, 1704-1712.

4. Coquerelle C, Oldenhove G, Acolty V, Denoeud J, Vansanten G, Verdebout J-M, Mellor A et al.: AntiCTLA-4 treatment induces IL-10-producing $\mathrm{ICOS}^{+}$regulatory $\mathrm{T}$ cells displaying IDO-dependent anti-inflammatory properties in a mouse model of colitis. Gut, 2009, 58, 1363-1373.

5. Engel MA, Neurath MF: New pathophysiological insights and modern treatment of IBD. J Gastroenterol, 2010, 45, 571-583. 
6. Hanauer SB: Inflammatory bowel disease: epidemiology, pathogenesis, and therapeutic opportunities. Inflam Bowel Dis, 2006, 12, Suppl 1, 3-9.

7. Jurynczyk M, Walczak A, Jurewicz A, JesionekKupnicka D, Szczepanik M, Selmaj K: Immune regulation of multiple sclerosis by transdermally applied myelin peptides. Ann Neurol, 2010, 68, 593-601.

8. Majewska M, Zając K, Kubera M, Bryniarski K, Ptak M, Basta-Kaim A, Książek L et al.: Effect of ovoalbumin on the survival of an $\mathrm{H}-\mathrm{Y}$ incompatible skin graft in C57BL/6 mice. Pharmacol Rep, 2006, 58, 439-442.

9. Majewska M, Zając K, Srebro Z, Sura P, Książek L, Zemelka M, Szczepanik M: Epicutaneous immunization with myelin basic protein protects from the experimental autoimmune encephalomyelitis. Pharmacol Rep, 2007, 59, 74-79.

10. Majewska-Szczepanik M, Strzępa A, Dorożyńska I, Motyl S, Banach T, Szczepanik M: Epicutaneous immunization with hapten-conjugated protein antigen alleviates contact sensitivity mediated by three different types of effector cells. Pharmacol Rep, 2012, 64, 919-926.

11. Majewska-Szczepanik M, Zemelka-Wiacek M, Ptak W, Wen Li, Szczepanik M: Epicutaneous immunization with DNP-BSA induces $\mathrm{CD} 4^{+} \mathrm{CD} 25^{+}$Treg cells that inhibit Tc1-mediated CS. Immunol Cell Biol, 2012, 90, 784-795.

12. Mayer L: Evolving paradigms in the pathogenesis of IBD. J Gastroenterol, 2010, 45, 9-16.

13. Neurath MF, Fuss I, Kelsall BL, Stuber E, Strober W: Antibodies to interleukin 12 abrogate established experimental colitis in mice. J Exp Med, 1995, 182, 1281-1290.

14. Peters A, Lee Y, Kuchroo VK: The many faces of Th17 cells. Curr Opin Immunol, 2011, 23, 702-706.

15. Pithadia AB, Jain S: Treatment of inflammatory bowel disease (IBD). Pharmacol. Rep, 2001, 63, 629-642.

16. Ptak W, Majewska M, Bryniarski K, Ptak M, Lobo FM, Zając K, Askenase PW, Szczepanik M: Epicutaneous immunization with protein antigen in the presence of TLR4 ligand induces TCR $\alpha \beta^{+} \mathrm{CD} 4^{+} \mathrm{T}$ contrasuppressor cells that reverse skin induced suppression of Th1 mediated contact sensitivity. J Immunol, 2009, 182, 837-850.

17. Ptak W, Szczepanik M, Bryniarski K, Tutaj M, Ptak M: Epicutaneous application of protein antigens incorporated into cosmetic cream induces antigen-nonspecific unresponsiveness in mice and affects the cell-mediated immune response. Int Arch Allergy Immunol, 2002, 128, $8-14$.

18. Rutz S, Ouyang W: Regulation of interleukin-10 and interleukin-22 expression in T helper cells. Curr Opin Immunol, 2011, 23, 605-612.

19. Strober W: Why study animal models of IBD? Inflam Bowel Dis, 2008, 129-131.

20. Szczepanik M: Mechanisms of immunological tolerance to the antigens of the central nervous system. Skininduced tolerance as a new therapeutic concept. J Physiol Pharmacol, 2011, 62, 159-165.

21. Szczepanik M: Skin induced tolerance and its reversion by Toll-like receptor (TLR) ligands. Arch Immunol Ther Exp (Warsz), 2007, 55, 161-172.

22. Szczepanik M, Bryniarski K, Tutaj M, Ptak M, Skrzeczynska J, Askenase PW, Ptak W: Epicutaneous immunization induces $\alpha \beta$ T-cell receptor CD4 CD8 double-positive non-specific suppressor T cells that inhibit contact sensitivity via transforming growth factorbeta. Immunology, 2005, 115, 42-54.

23. Szczepanik M, Gryglewski A, Bryniarski K, Stachura J, Ptak W: Experimental inflammatory bowel disease - role of T cells. J Physiol Pharmacol, 2000, 51, 333-346.

24. Szczepanik M, Tutaj M, Bryniarski K, Dittel BN: Epicutaneously induced TGF- $\beta$-dependent tolerance inhibits experimental autoimmune encephalomyelitis. J Neuroimmunol, 2005, 164, 105-114.

25. Tutaj M, Szczepanik M: Epicutaneous (EC) immunization with myelin basic protein (MBP) induces TCR $\alpha \beta^{+}$ $\mathrm{CD}^{+} \mathrm{CD}^{+}$double positive suppressor cells that protect from experimental autoimmune encephalomyelitis (EAE). J Autoimmun, 2007, 28, 208-215.

26. Wirtz S, Neufert C,Weigmann B, Neurath MF: Chemically induced mouse models of intestinal inflammation. Nat Protoc, 2007, 2, 541-546.

27. Wirtz S, Neurath MF: Mouse models of inflammatory bowel disease. Adv Drug Deliv Rev, 2007, 59, 1073-1083.

28. Yang X, Meng S, Jiang H, Chen T, Wu W: Exosomes derived from interleukin-10-treated dendritic cells can inhibit trinitrobenzene sulfonic acid-induced rat colitis. Scand J Gastroenterol, 2010, 45, 1168-1177.

Received: March 2, 2012; in the revised form: September 4, 2012; accepted: September 25, 2012. 